\title{
The Crystal Structure of $\operatorname{LiNb}_{6} \mathbf{O}_{15} F$
}

\author{
MON I C A L U N D B ER G
}

Institute of Inorganic and Physical Chemistry, University of Stockholm, Stockholm, Sweden

The crystal structure of $\mathrm{LiNb}_{6} \mathrm{O}_{15} \mathrm{~F}$ has been determined from threedimensional single-crystal data. The orthorhombic unit-cell dimensions are:

$$
a=16.635 \AA ; b=3.964 \AA ; c=8.888 \AA
$$

The space group is Pmma. The structure can be described as being built up of blocks containing one pentagonal bipyramid sharing edges with five octahedra. These building blocks are joined together by having corners in common.

The results of a phase analysis of the system $\mathrm{LiF}-\mathrm{Nb}_{2} \mathrm{O}_{5}$ were reported in a 1 recent publication. ${ }^{1}$ Two oxide fluoride phases were found, viz. $\mathrm{LiNb}_{6} \mathrm{O}_{15} \mathrm{~F}$ and $\mathrm{LiF} \cdot 10-12 \mathrm{Nb}_{2} \mathrm{O}_{5}$. The crystal structure of the former has now been determined and will be described below.

\section{EXPERIMENTAL}

The compound $\mathrm{LiNb}_{6} \mathrm{O}_{15} \mathrm{~F}$ was prepared by heating a mixture of $\mathrm{LiF}$ (Baker's Analyzed, $99.89 \%$ ) and $\alpha-\mathrm{Nb}_{2} \mathrm{O}_{5}$ (Kawecki, 99.99\%), in the mole ratio $1: 3$, in a sealed platinum tube at $1200^{\circ} \mathrm{C}$ for two days applying a technique developed by Roth. ${ }^{2}$ The product consisted of colourless, rod-shaped crystals up to $0.5 \mathrm{~mm}$ in length. The singlecrystal X-ray examination showed the crystals to be of orthorhombic symmetry. From the indexed Guinier X-ray powder pattern, given in Table 1, the following unit-cell dimensions were derived:

$$
a=16.635 \AA ; b=3.964 \AA ; c=8.888 \AA
$$

The crystal was rotated about the rod axis and the $h 0 l, h 1 l$, and $h 2 l$ reflections were collected in an integrating Weissenberg camera using filtered $\mathrm{Cu} K$ radiation. The crystal was $0.19 \mathrm{~mm}$ in length and had the cross section edges 0.03 and $0.01 \mathrm{~mm}$. The reflections were registered by the multiple-film technique and visually estimated by means of a standard scale. The data were corrected for absorption using a program written by Werner $^{3}$ for the computer FACIT EDB.

\section{STRUCTURE DETERMINATION}

The calculated density $4.67 \mathrm{~g} / \mathrm{cm}^{3}$ with two formula units $\mathrm{LiNb}_{6} \mathrm{O}_{15} \mathrm{~F}$ per unit cell, agrees well with the observed density, $4.62 \mathrm{~g} / \mathrm{cm}^{3}$. The only sys- 
Table 1. Guinier X-ray powder pattern of $\mathrm{LiNb}_{6} \mathrm{O}_{15} \mathrm{~F}$, internally calibrated with $\mathrm{KCl}$.

\begin{tabular}{llll}
\hline & $\sin ^{2} \Theta_{\text {obs }}$ & $h k l$ & $\sin ^{2} \Theta_{\text {calc }}$ \\
\hline w & & & \\
m & 0.00751 & 001 & 0.00751 \\
w & 0.00858 & 200 & 0.00858 \\
w & 0.00969 & 101 & 0.00968 \\
w & 0.02680 & 301 & 0.02680 \\
w & 0.03004 & 002 & 0.03004 \\
wst & 0.03217 & 102 & 0.03218 \\
w & 0.03775 & 010 & 0.03774 \\
w & 0.03862 & 202 & 0.03862 \\
st & 0.04184 & 401 & 0.04181 \\
st & 0.04631 & 210 & 0.04632 \\
vw & 0.04933 & 302 & 0.04934 \\
vw & 0.06112 & 501 & 0.06111 \\
m & 0.06446 & 311 & 0.06454 \\
m & 0.06776 & 012 & 0,06778 \\
m & 0,06972 & 103 & 0.06973 \\
m & 0.07616 & 203 & 0.07617 \\
w & 0.07716 & 600 & 0.07718 \\
w & 0.07955 & 411 & 0.07955 \\
st & 0.08363 & 502 & 0.08364 \\
m & 0.08467 & 601 & 0.08469 \\
w & 0.08709 & $\mathbf{3 1 2}$ & 0.08708 \\
m & 0.09883 & 511 & 0.09885 \\
st & 0.10199 & 412 & 0.10208 \\
& 0.10731 & 602 & 0.10722 \\
& 0.15091 & 020 & 0.15096 \\
\hline
\end{tabular}

tematically absent reflections are $h k 0$, when $h$ is odd. This is characteristic of the space groups $P 2_{1} m a$ (No. 26), Pm2a (No. 28), and Pmma (No. 51).

The $h 0 l, h 1 l$, and $h 2 l$ layer lines, with due regard for observational errors and for the effects of thermal motion, were found to be very similar. This

Table 2. Atomic parameters in $\mathrm{LiNb}_{6} \mathrm{O}_{15} \mathrm{~F}$. Space group Pmma.

\begin{tabular}{|c|c|c|c|c|c|c|}
\hline Atom & Position & $x$ & $y$ & $z$ & $B$ & $\sigma B$ \\
\hline $\begin{array}{l}2 \mathrm{Nb}_{1} \\
4 \mathrm{Nb}_{2} \\
4 \mathrm{Nb}_{3} \\
2 \mathrm{Nb}_{4} \\
2 \mathrm{O}_{1} \\
4 \mathrm{O}_{2} \\
4 \mathrm{O}_{3} \\
2 \mathrm{O}_{8} \\
4 \mathrm{O}_{8} \\
4 \mathrm{O}_{8} \\
4 \mathrm{O}_{7} \\
2 \mathrm{O}_{8} \\
4 \mathrm{O}_{8} \\
2 \mathrm{O}_{10}\end{array}$ & $\begin{array}{r}2(e) \\
4(i) \\
4(i) \\
2(e) \\
2(e) \\
4(i) \\
4(i) \\
\text { F) } 2(f) \\
4(i) \\
4(i) \\
4(j) \\
2(a) \\
4(j) \\
2(f)\end{array}$ & 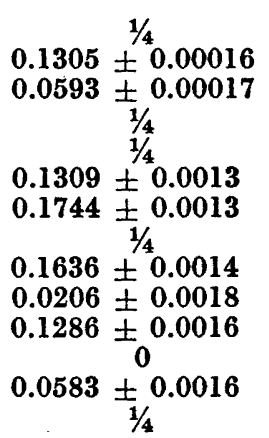 & $\begin{array}{l}0 \\
0 \\
0 \\
0 \\
0 \\
0 \\
0 \\
1 / 2 \\
0 \\
0 \\
11 / 2 \\
0 \\
1 / 2 \\
1 / 2\end{array}$ & $\begin{array}{l}0.6874 \pm 0.0004 \\
0.3973 \pm 0.0003 \\
0.8146 \pm 0.0003 \\
0.0578 \pm 0.0005 \\
0.4582 \pm 0.0042 \\
0.6149 \pm 0.0023 \\
0.8823 \pm 0.0022 \\
0.6922 \pm 0.0032 \\
0.1963 \pm 0.0023 \\
0.3386 \pm 0.0030 \\
0.3998 \pm 0.0029 \\
0.8170 \pm 0.0027 \\
0.0524 \pm 0.0043\end{array}$ & $\begin{array}{l}0.49 \\
0.54 \\
0.52 \\
0.69 \\
1.3 \\
0.3 \\
0 \\
0.2 \\
0.5 \\
1.2 \\
1.5 \\
1.2 \\
1.0 \\
1.5\end{array}$ & $\begin{array}{l}0.07 \\
0.05 \\
0.05 \\
0.07 \\
0.7 \\
0.4 \\
0.4 \\
0.6 \\
0.4 \\
0.5 \\
0.5 \\
1.0 \\
0.5 \\
0.7\end{array}$ \\
\hline
\end{tabular}

Acta Chem. Scand. 19 (1965) No. 10 


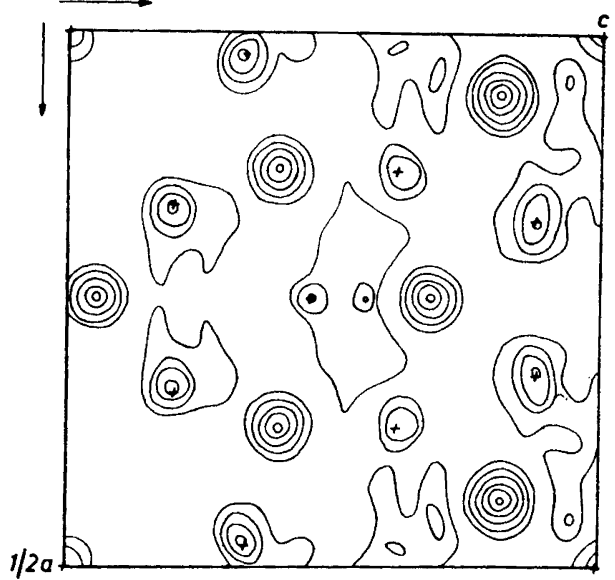

Fig. 1. Electron density projection along [010]. The figure is on an arbitrary scale and only every third contour is drawn for the niobium peak.

suggests that all the niobium atoms are situated in one plane, and rather likely a mirror plane, perpendicular to the $b$-axis. The space group with the highest symmetry, Pmma, comprises the following point positions in such planes of symmetry:

$$
\begin{aligned}
& \text { 4(j) } m \quad x, \frac{1}{2}, z ; \bar{x}, \frac{1}{2}, \bar{z} ; \frac{1}{2}+x, \frac{1}{2}, \bar{z} ; \frac{1}{2}-x, \frac{1}{2}, z . \\
& \text { 4(i) } m \quad x, 0, z ; \bar{x}, 0, \bar{z} ; \frac{1}{2}+x, 0, \bar{z} ; \frac{1}{2}-x, 0, z . \\
& 2(f) m m \quad \frac{1}{4}, \frac{1}{2}, z ; \frac{3}{4}, \frac{1}{2}, \bar{z} . \\
& 2(e) m m \quad \frac{1}{4}, 0, z ; \frac{3}{4}, 0, \bar{z} . \\
& 2(d) 2 / m 0, \frac{1}{2}, \frac{1}{2} ; \frac{1}{2}, \frac{1}{2}, \frac{1}{2} . \\
& 2(c) 2 / m 0,0, \frac{1}{2} ; \frac{1}{2}, 0, \frac{1}{2} . \\
& 2(b) 2 / m 0, \frac{1}{2}, 0 ; \frac{1}{2}, \frac{1}{2}, 0 . \\
& 2(a) 2 / m 0,0,0 ; \frac{1}{2}, 0,0 .
\end{aligned}
$$

Using the Patterson projection along [010], eight niobium atoms were placed in two $4(i)$ positions and the other four niobium atoms in two $2(e)$ positions. The corresponding electron density projection (Fig. 1) showed all the heavy atoms in the expected positions.

In addition to the high niobium atom maxima the electron density projection was found to contain several lower ones likely to correspond to non-metal atoms (cf. Fig. 1). The distribution of these minor peaks is a fivefold one around one of the metal atoms of one of the $2(e)$ positions and roughly square around the other niobium atoms. This suggested that the non-metal coordination around the former is a pentagonal bipyramid with non-metal atoms, not disclosed in the map overlapping the niobium atoms. The arrangement around all the other metal atoms would by analogy be an octahedron of anions. In this way sixteen anion atoms were placed in four $4(i)$ positions, two in $2(e)$ and two in 2(a), all at the same $y$ level as the metal atoms. The twelve top 
anions of the polyhedra were placed in two $(4 j)$ and two $2(f)$ positions with $x$ and $z$ parameters equal to those of the metal atoms.

This trial structure was refined by means of successive $F_{\mathrm{o}}-F_{\mathrm{c}}$ Fourier summations until the reliability factor $R$ was 0.135 . The final $F_{\mathrm{o}}^{\circ}-F_{\mathrm{c}}^{\mathrm{c}}$ summation led to no shifts of the atoms and the general shape of this synthesis was fairly smooth.

Assuming $P m m a$ to be the correct space group, 409 independent reflections from $h 0 l, h 1 l$, and $h 2 l$ were finally refined by the least-squares method using the Åsbrink and Brändén ${ }^{4}$ program written for FACIT. The refinement was stopped when the shifts of the positional parameters were less than $0.5 \%$ of the standard deviations. The $R$ factor was then 0.091 .

During the determination and refinement of the structure all the non-metal atoms were given the form factor of an oxygen, which very nearly corresponds to a random distribution of oxygen and fluorine on the anion sites. No account was taken of the arrangement of the two lithium atoms in the unit cell.

Atomic coordinates and temperature factors with their standard deviations are given in Table 2. The comparison between observed and calculated structure factors is presented in Table 3.

\section{DESCRIPTION AND DISCUSSION OF THE STRUCTURE}

The structure of $\mathrm{LiNb}_{6} \mathrm{O}_{15} \mathrm{~F}$ illustrated in Fig. 2 can be described as a polygonal network which contains identical building blocks each consisting of one pentagonal bipyramid sharing edges with five octahedra. The building blocks are joined together with corners in common in the $b$-direction as well as in the $a c$-plane. The $b$-axis of the unit-cell corresponds to the space-diagonal of the polyhedra.
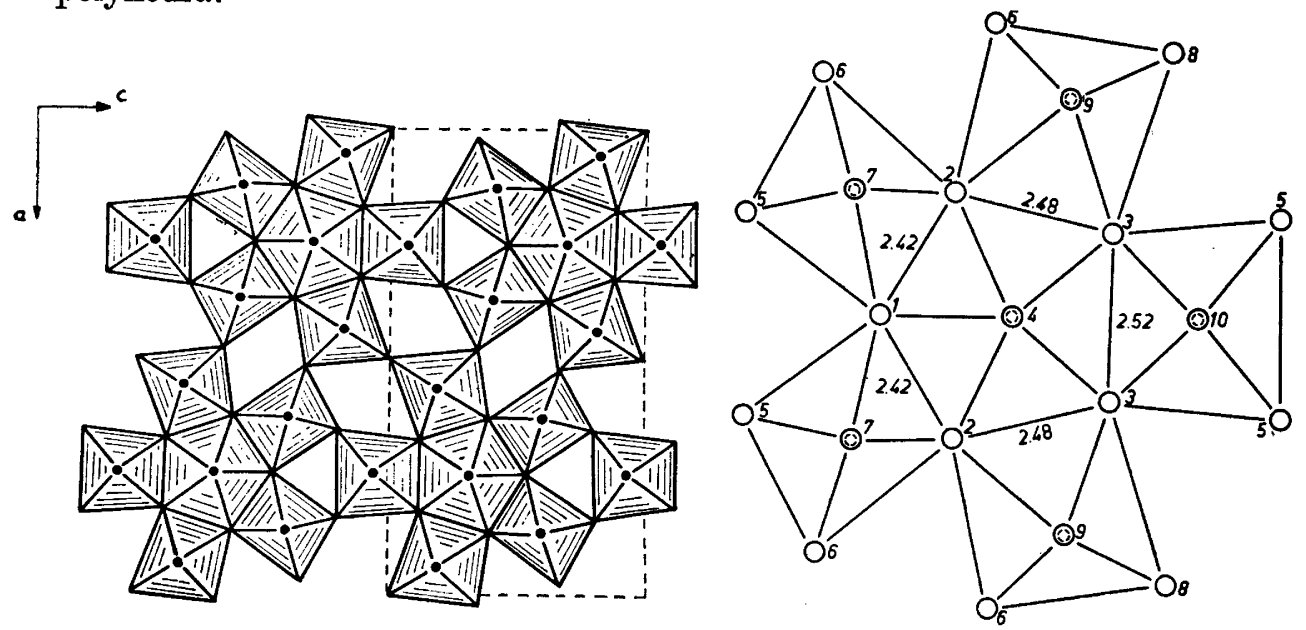

Fig. 2. The crystal structure of $\mathrm{LiNb}_{6} \mathrm{O}_{15} \mathrm{~F}$. Fig. 3. The pentagonally bipyramidal coordination in $\mathrm{LiNb}_{6} \mathrm{O}_{15} \mathrm{~F}$. $\mathrm{Nb}_{1}$ is situated in the center of the polyhedron. Small circle represents niobium, large circle represents oxygen.

Acta Chem. Scand. 19 (1965) No. 10 
Table 3. Observed and calculated $\boldsymbol{F}$-values.

\begin{tabular}{|c|c|c|c|c|c|c|c|}
\hline$h k$ & $k l$ & $F_{\text {obs }}$ & $\boldsymbol{F}_{\text {calc }}$ & $h \quad k$ & $l$ & $\boldsymbol{F}_{\mathrm{obs}}$ & $\boldsymbol{F}_{\text {calc }}$ \\
\hline 20 & 00 & - & 63 & 60 & 3 & $<26$ & 4 \\
\hline 40 & 00 & 20 & 14 & 70 & 3 & 155 & 138 \\
\hline 60 & 00 & 187 & 147 & 80 & 3 & 302 & 267 \\
\hline 80 & 00 & 192 & 158 & 90 & 3 & 165 & 157 \\
\hline 100 & 00 & 288 & 271 & 100 & 3 & $<\mathbf{3 3}$ & 16 \\
\hline 120 & 00 & $<34$ & 5 & 110 & 3 & 60 & 49 \\
\hline 140 & 00 & $<37$ & 16 & 120 & 3 & 36 & 42 \\
\hline 160 & $\begin{array}{ll}0 & 0\end{array}$ & 244 & 238 & 130 & 3 & 53 & 54 \\
\hline 180 & 00 & 48 & 42 & 140 & 3 & 84 & 73 \\
\hline 200 & 00 & 26 & 29 & 150 & 3 & 92 & 89 \\
\hline 00 & 01 & - & 29 & 160 & 03 & $<37$ & 14 \\
\hline 10 & $\begin{array}{ll}0 & 1\end{array}$ & 37 & 35 & 170 & 3 & 102 & 114 \\
\hline 20 & 01 & $<14$ & 9 & 180 & 03 & 113 & 132 \\
\hline 30 & 01 & $\mathbf{3 3}$ & 28 & 00 & 4 & 143 & 149 \\
\hline 40 & 01 & 205 & 178 & 10 & 4 & 24 & 29 \\
\hline 50 & 01 & 266 & 242 & 20 & 4 & $<25$ & 4 \\
\hline 60 & 01 & 75 & 65 & 30 & 4 & 79 & 92 \\
\hline 70 & $\begin{array}{ll}0 & 1\end{array}$ & 58 & 53 & 40 & 04 & 131 & 142 \\
\hline 80 & 01 & 163 & 151 & 50 & 04 & 172 & 191 \\
\hline 90 & 01 & 71 & 65 & 60 & 04 & 50 & 42 \\
\hline 100 & 01 & 30 & 30 & 70 & 04 & 98 & 96 \\
\hline 110 & 01 & 153 & 141 & 80 & 34 & 81 & 75 \\
\hline 120 & 01 & 121 & 113 & 90 & 04 & 66 & 71 \\
\hline 130 & 01 & 36 & 21 & 100 & 04 & 35 & 29 \\
\hline 140 & 01 & 37 & 41 & 110 & 04 & 76 & 74 \\
\hline 150 & $\begin{array}{ll}0 & 1\end{array}$ & 84 & 80 & 120 & 04 & 91 & 88 \\
\hline 160 & 01 & $<38$ & 8 & 130 & ] 4 & 65 & 54 \\
\hline 170 & 01 & $<36$ & 12 & 140 & 04 & 38 & 32 \\
\hline 180 & 01 & $\mathbf{5 3}$ & 58 & 150 & 4 & 36 & 31 \\
\hline 190 & 01 & $<\mathbf{3 0}$ & 17 & 160 & 4 & 49 & 58 \\
\hline 200 & 01 & 54 & 69 & 170 & 4 & 32 & 29 \\
\hline 00 & 02 & 61 & 67 & 180 & 4 & 28 & 28 \\
\hline 10 & 02 & 38 & 47 & 190 & 4 & 37 & 47 \\
\hline 20 & 02 & 84 & 92 & 00 & 5 & 169 & 179 \\
\hline 30 & 02 & 277 & 294 & 10 & 05 & 114 & 113 \\
\hline 40 & 02 & 28 & 30 & 20 & 5 & 140 & 150 \\
\hline 50 & 02 & 93 & 80 & 30 & 5 & 41 & 45 \\
\hline 60 & 02 & 43 & 47 & 40 & 5 & 184 & 195 \\
\hline 70 & 02 & 113 & 104 & 50 & 5 & 107 & 122 \\
\hline 80 & 02 & 170 & 140 & 60 & 5 & 32 & 35 \\
\hline 90 & 02 & $<29$ & 5 & 70 & 5 & 58 & 53 \\
\hline 100 & 02 & 73 & 62 & 80 & 05 & 34 & 36 \\
\hline 110 & 02 & 57 & 57 & 90 & 5 & 50 & 48 \\
\hline 120 & 02 & $<35$ & 8 & 100 & 5 & 52 & 52 \\
\hline 130 & 02 & 201 & 193 & 110 & 5 & 115 & 106 \\
\hline 140 & 02 & $<38$ & 15 & 120 & 5 & 139 & 155 \\
\hline 150 & 02 & $<\mathbf{3 8}$ & 7 & 130 & 5 & $<38$ & 21 \\
\hline 160 & 02 & $<37$ & 21 & 140 & 5 & 115 & 128 \\
\hline 170 & 02 & $<36$ & 22 & 150 & 05 & 77 & 81 \\
\hline 180 & 02 & 51 & 58 & 160 & 5 & 68 & 81 \\
\hline 00 & 03 & 20 & 20 & 170 & 05 & 40 & 41 \\
\hline 10 & 03 & 149 & 187 & 180 & 05 & 45 & 60 \\
\hline 20 & 03 & 120 & 155 & 00 & 06 & 32 & 25 \\
\hline 30 & 03 & 22 & 22 & 10 & 06 & 130 & 118 \\
\hline 0 & 03 & 65 & 64 & 20 & 6 & 73 & 75 \\
\hline 50 & 03 & 69 & 73 & 30 & 06 & 102 & 103 \\
\hline
\end{tabular}


STRUCTURE OF $\operatorname{LiNb}_{6} \mathrm{O}_{15} F$

\begin{tabular}{|c|c|c|c|c|c|c|c|c|}
\hline$h$ & $l \quad l$ & $\boldsymbol{F}_{\text {obs }}$ & $F_{\text {calc }}$ & $h$ & $k$ & $l$ & $F_{\text {obs }}$ & $F_{\text {calc }}$ \\
\hline 4 & 6 & 107 & 114 & 1 & $\begin{array}{ll}01 \\
1\end{array}$ & & 31 & 31 \\
\hline 5 & 6 & 60 & 53 & 2 & 01 & 0 & 31 & 25 \\
\hline 6 & 6 & 50 & 58 & 3 & 01 & 0 & 99 & 91 \\
\hline 7 & 6 & 111 & 112 & 4 & 01 & & 87 & 78 \\
\hline 8 & b 6 & 130 & 126 & 5 & $\begin{array}{lll}0 & 1\end{array}$ & & 28 & 23 \\
\hline 9 & 06 & 115 & 121 & 6 & 01 & 10 & $<28$ & 4 \\
\hline 10 & $0 \quad 6$ & 38 & 40 & 7 & 01 & 10 & 66 & 64 \\
\hline 11 & 6 & $<\mathbf{3 8}$ & 8 & 2 & 1 & 0 & 49 & 40 \\
\hline 12 & D 6 & 52 & 56 & 4 & 1 & 0 & $<18$ & 7 \\
\hline 13 & j 6 & 71 & 78 & 6 & 1 & 0 & 85 & 79 \\
\hline 14 & 06 & $<34$ & 3 & 8 & 1 & 0 & 115 & 113 \\
\hline 15 & $\begin{array}{ll}0 & 6\end{array}$ & 31 & 33 & 10 & 1 & 0 & 196 & 202 \\
\hline 16 & 0 6 & $<28$ & 9 & 12 & 1 & 0 & $<31$ & 8 \\
\hline 17 & $0 \quad 6$ & 55 & 76 & 14 & 1 & 0 & $<33$ & 9 \\
\hline 0 & $\begin{array}{l}0 \\
0\end{array}$ & 36 & 34 & 16 & 1 & 0 & 170 & 188 \\
\hline 1 & 07 & 102 & 94 & 18 & 1 & 0 & 40 & 37 \\
\hline$\overline{2}$ & 07 & $<36$ & 5 & & 1 & 1 & 14 & 20 \\
\hline $\mathbf{3}$ & 07 & 134 & 120 & 1 & 1 & l & 24 & 20 \\
\hline 4 & 07 & 37 & 35 & 2 & 1 & 1 & $<13$ & 6 \\
\hline 5 & 07 & 37 & 45 & 3 & 1 & 1 & 27 & 19 \\
\hline 6 & 07 & 37 & 33 & 4 & 1 & 1 & 112 & 111 \\
\hline 7 & $\begin{array}{ll}0 & 7\end{array}$ & 38 & 28 & 5 & 1 & 1 & 151 & 152 \\
\hline 8 & 07 & 38 & 24 & 6 & 1 & 1 & 49 & 39 \\
\hline 9 & 07 & 65 & 58 & 7 & 1 & i & 34 & 31 \\
\hline 10 & 07 & 37 & 35 & 8 & 1 & 1 & 119 & 111 \\
\hline 11 & 07 & 36 & 42 & 9 & i & 1 & 60 & 52 \\
\hline 12 & $\begin{array}{l}0 \\
0\end{array}$ & $<35$ & 18 & 10 & 1 & 1 & $<27$ & 16 \\
\hline 13 & 0 7 & 141 & 127 & 11 & 1 & I & 113 & 102 \\
\hline 14 & 07 & $<\mathbf{3 0}$ & 9 & 12 & i & i & 95 & 89 \\
\hline 15 & 07 & 58 & 72 & 13 & 1 & 1 & 32 & 18 \\
\hline 0 & $\begin{array}{ll}0 & 8\end{array}$ & 136 & 129 & 14 & 1 & 1 & 33 & 35 \\
\hline 1 & $\begin{array}{ll}0 & 8\end{array}$ & 53 & 56 & 15 & 1 & 1 & 66 & 64 \\
\hline$\overline{2}$ & ] 8 & $<38$ & 21 & 16 & 1 & 1 & $<33$ & 6 \\
\hline $\mathbf{3}$ & $\begin{array}{ll}0 & 8\end{array}$ & $<\mathbf{3 8}$ & 27 & 17 & i & i & $<\mathbf{3 1}$ & 11 \\
\hline 4 & $\begin{array}{ll}0 & 8\end{array}$ & 151 & 131 & 18 & i & I & 44 & 50 \\
\hline$\overline{5}$ & 08 & 65 & 64 & 19 & 1 & 1 & $<25$ & 17 \\
\hline 6 & $\begin{array}{l}0 \\
0\end{array}$ & 152 & 142 & 20 & 1 & 1 & 47 & 58 \\
\hline 7 & $\begin{array}{ll}0 & 8\end{array}$ & 52 & 51 & 0 & 1 & 2 & 43 & 43 \\
\hline 8 & 08 & 36 & 36 & 1 & 1 & 2 & 26 & 29 \\
\hline 9 & $\begin{array}{ll}0 & 8\end{array}$ & 70 & 56 & 2 & 1 & 2 & 51 & 60 \\
\hline 10 & 0 & 135 & 129 & 3 & 1 & 2 & 158 & 183 \\
\hline 11 & $\begin{array}{ll}0 & 8\end{array}$ & $<32$ & 22 & 4 & 1 & 2 & $<18$ & 13 \\
\hline 12 & 08 & 84 & 79 & 5 & 1 & $\overline{2}$ & 54 & 50 \\
\hline 13 & $\begin{array}{ll}0 & 8\end{array}$ & 46 & 43 & 6 & l & 2 & 22 & 29 \\
\hline 14 & $\begin{array}{ll}0 & 8\end{array}$ & 56 & 46 & 7 & $\hat{\mathbf{l}}$ & 2 & 85 & 79 \\
\hline 0 & $\begin{array}{ll}0 & 9\end{array}$ & 118 & 119 & 8 & 1 & 2 & 114 & 102 \\
\hline 1 & $\begin{array}{ll}0 & 9\end{array}$ & $<\mathbf{3 7}$ & 24 & 9 & 1 & 2 & $<27$ & 5 \\
\hline 2 & $\begin{array}{ll}0 & 9\end{array}$ & $<\mathbf{3 7}$ & 1 & 10 & 1 & 2 & 53 & 45 \\
\hline $\mathbf{3}$ & $\begin{array}{ll}0 & 9\end{array}$ & $<\mathbf{3 7}$ & 2 & 11 & 1 & 2 & 42 & 41 \\
\hline 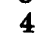 & $\begin{array}{ll}0 & 9\end{array}$ & 71 & 57 & 12 & l & 2 & $<32$ & 5 \\
\hline 5 & $\begin{array}{ll}0 & 9\end{array}$ & 156 & 137 & 13 & 1 & 2 & 139 & 146 \\
\hline 6 & $\begin{array}{ll}0 & 9\end{array}$ & & 41 & 14 & 1 & 2 & $<33$ & 13 \\
\hline 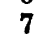 & $\begin{array}{ll}0 & 9\end{array}$ & $<34$ & 11 & 15 & l & 2 & $<\mathbf{3 3}$ & 3 \\
\hline 8 & $\begin{array}{ll}0 & 9\end{array}$ & 55 & 50 & 16 & 1 & 2 & $<32$ & 14 \\
\hline 9 & $\begin{array}{ll}0 & 9\end{array}$ & $<\mathbf{3 1}$ & 9 & 17 & 1 & 2 & $<\mathbf{3 0}$ & 21 \\
\hline 10 & $\begin{array}{ll}0 & 9\end{array}$ & 71 & 71 & 18 & 1 & 2 & 43 & 49 \\
\hline 11 & $\begin{array}{ll}0 & 9\end{array}$ & 71 & 82 & 0 & 1 & 3 & 18 & 17 \\
\hline 0 & $\begin{array}{lll}0 & 10\end{array}$ & 130 & 121 & 1 & 1 & 3 & 85 & 113 \\
\hline
\end{tabular}

Acta Chem. Scand. 19 (1965) No. 10 
MONICA L N D B R G

\begin{tabular}{|c|c|c|c|c|c|c|c|}
\hline$h k$ & $l$ & $F_{\text {obs }}$ & $F_{\text {calc }}$ & $h k$ & $l$ & $F_{\text {obs }}$ & $\boldsymbol{F}_{\text {calc }}$ \\
\hline 21 & 3 & 68 & 89 & 21 & 6 & 66 & 58 \\
\hline 31 & 3 & $<20$ & 13 & 31 & 6 & 79 & 75 \\
\hline 41 & $\mathbf{3}$ & 42 & 46 & 41 & 6 & 91 & 87 \\
\hline 51 & $\mathbf{3}$ & 59 & 57 & 51 & 6 & 44 & 47 \\
\hline 61 & $\mathbf{3}$ & $<24$ & 1 & 61 & 6 & 44 & 43 \\
\hline 71 & $\mathbf{3}$ & 105 & 98 & 71 & 6 & 82 & 80 \\
\hline 81 & 3 & 189 & 198 & 81 & 6 & 95 & 95 \\
\hline 91 & 3 & 119 & 115 & 91 & 6 & 96 & 93 \\
\hline 101 & 3 & $<30$ & 14 & 101 & 6 & $<33$ & 26 \\
\hline 111 & $\mathbf{3}$ & 44 & 39 & 111 & 6 & $<33$ & 5 \\
\hline 121 & 3 & 32 & 27 & 121 & 6 & 45 & 45 \\
\hline 131 & 3 & 46 & 45 & 131 & 6 & 61 & 62 \\
\hline 141 & 3 & 57 & 53 & 141 & 6 & $<29$ & 2 \\
\hline 151 & 3 & 65 & 67 & 151 & 6 & 26 & 24 \\
\hline 161 & 3 & $<31$ & 11 & 161 & 6 & $<23$ & 5 \\
\hline 171 & 3 & 84 & 93 & 171 & 6 & 53 & 60 \\
\hline 181 & 3 & 97 & 107 & 01 & 7 & 45 & 34 \\
\hline 01 & 4 & 100 & 111 & 11 & 7 & 72 & 69 \\
\hline 11 & 4 & 22 & 22 & 21 & 7 & $<32$ & 2 \\
\hline 21 & 4 & $<23$ & 4 & 31 & 7 & 97 & 91 \\
\hline 31 & 4 & 62 & 67 & 41 & 7 & 32 & 27 \\
\hline 41 & 4 & 97 & 104 & 51 & 7 & 33 & 35 \\
\hline 51 & 4 & 124 & 139 & 61 & 7 & 33 & 29 \\
\hline 61 & 4 & 37 & 34 & 71 & 7 & 33 & 24 \\
\hline 71 & 4 & 65 & 66 & 81 & 7 & 33 & 15 \\
\hline 81 & 4 & 61 & 59 & 91 & 7 & 46 & 45 \\
\hline 91 & 4 & 52 & 52 & 101 & 7 & 32 & 32 \\
\hline 101 & 4 & 31 & 25 & 111 & 7 & 31 & 33 \\
\hline 111 & 4 & 56 & 53 & 121 & 7 & $<29$ & 16 \\
\hline 121 & 4 & 66 & 69 & 131 & 7 & 111 & 106 \\
\hline 131 & 4 & 33 & 41 & 141 & 7 & $<25$ & 9 \\
\hline 141 & 4 & 32 & 26 & 151 & 7 & 53 & 56 \\
\hline 151 & 4 & 31 & 27 & $\begin{array}{ll}0 & 1\end{array}$ & 8 & 112 & 96 \\
\hline 161 & 4 & 41 & 48 & 11 & 8 & 57 & 45 \\
\hline 171 & 4 & 27 & 19 & 21 & 8 & $<\mathbf{3 3}$ & 16 \\
\hline 181 & 4 & 23 & 27 & $\begin{array}{ll}3 & 1\end{array}$ & 8 & $<\mathbf{3 3}$ & 22 \\
\hline 191 & 4 & 28 & 39 & 41 & 8 & 125 & 105 \\
\hline $\begin{array}{ll}0 & 1\end{array}$ & 5 & 120 & 133 & 51 & 8 & 56 & 48 \\
\hline 11 & 5 & 84 & 83 & 61 & 8 & 129 & 113 \\
\hline 21 & 5 & 103 & 107 & 71 & 8 & 45 & 40 \\
\hline $\begin{array}{ll}3 & 1\end{array}$ & 5 & 46 & 39 & 81 & 8 & 31 & 32 \\
\hline 41 & 5 & 128 & 146 & 91 & 8 & 51 & 45 \\
\hline 51 & 5 & 89 & 90 & 101 & 8 & 119 & 102 \\
\hline 61 & $\mathbf{5}$ & 29 & 28 & 111 & 8 & $<27$ & 15 \\
\hline 71 & 5 & 30 & 43 & 121 & 8 & 73 & 66 \\
\hline 81 & 5 & 31 & 23 & 131 & 8 & 37 & 37 \\
\hline 91 & 5 & 32 & 36 & 141 & 8 & 30 & 38 \\
\hline 101 & 5 & 32 & 45 & 01 & 9 & 110 & 93 \\
\hline 111 & 5 & 90 & 86 & 11 & 9 & $<32$ & 15 \\
\hline 121 & 5 & 112 & 121 & 21 & 9 & & 1 \\
\hline 131 & $\mathbf{5}$ & $<33$ & 19 & 31 & 9 & $<31$ & 6 \\
\hline 141 & 5 & 103 & 106 & 41 & 9 & 52 & 51 \\
\hline 151 & $\mathbf{5}$ & 65 & 66 & 51 & 9 & 122 & 109 \\
\hline 161 & 5 & 68 & 66 & 61 & 9 & 41 & 32 \\
\hline 171 & 5 & 33 & 33 & 71 & 9 & $<28$ & 10 \\
\hline 181 & 5 & 35 & 45 & 81 & 9 & 49 & 43 \\
\hline 01 & 6 & 29 & 20 & 01 & 10 & 101 & 98 \\
\hline 11 & 6 & 95 & 88 & 11 & 10 & 26 & 28 \\
\hline
\end{tabular}

Acta Chem. Scand. 19 (1965) No. 10 
STR U CTURE OF $\operatorname{LiNb}_{6} \mathrm{O}_{15} \mathrm{~F}$

\begin{tabular}{|c|c|c|c|c|c|c|c|c|}
\hline$h$ & $k l$ & & $F_{\text {obs }}$ & $F_{\text {calc }}$ & $h k$ & & $F_{\text {obs }}$ & $F_{\text {calc }}$ \\
\hline 2 & 110 & 0 & 25 & 22 & 52 & 3 & 53 & 50 \\
\hline 3 & 110 & 0 & 79 & 73 & 62 & 3 & $<22$ & 4 \\
\hline 4 & 110 & 0 & 77 & 68 & 72 & 3 & 117 & 109 \\
\hline 5 & 110 & 0 & 24 & 18 & 82 & 3 & 228 & 217 \\
\hline 6 & 110 & 0 & $<23$ & 6 & 92 & 3 & 133 & 130 \\
\hline 7 & 110 & 0 & 62 & 54 & 102 & 3 & $<27$ & 11 \\
\hline 2 & 20 & 0 & 49 & 45 & 112 & 3 & 51 & 42 \\
\hline 4 & 2 & 0 & $<18$ & 10 & 122 & 3 & 28 & 38 \\
\hline 6 & 2 & 0 & 147 & 125 & 132 & 3 & 39 & 48 \\
\hline 8 & 2 & 0 & 134 & 121 & 142 & 3 & 59 & 65 \\
\hline 10 & 2 & 0 & 228 & 223 & 152 & 3 & 71 & 79 \\
\hline 12. & 2 & 0 & $<27$ & 3 & 162 & $\mathbf{3}$ & $<75$ & 12 \\
\hline 14 & 2 & 0 & $<28$ & 14 & 172 & $\mathbf{3}$ & 93 & 101 \\
\hline 16 & 2 & 0 & 190 & 210 & 182 & $\mathbf{3}$ & 98 & 127 \\
\hline 18 & 2 & 0 & 32 & 37 & 02 & 4 & 105 & 114 \\
\hline 0 & 2 & 1 & 14 & 21 & 12 & 4 & 21 & 20 \\
\hline 1 & 21 & 1 & 28 & 27 & 22 & 4 & $<22$ & 3 \\
\hline 2 & 21 & 1 & 12 & 8 & 32 & 4 & 74 & 73 \\
\hline 3 & 21 & 1 & 26 & 20 & 42 & 4 & 106 & 111 \\
\hline 4 & 21 & 1 & 149 & 133 & 52 & 4 & 143 & 152 \\
\hline 5 & 21 & 1 & 220 & 188 & 62 & 4 & 34 & 32 \\
\hline 6 & 21 & 1 & 61 & 56 & 72 & 4 & 93 & 80 \\
\hline 7 & 21 & 1 & 46 & 44 & 82 & 4 & 55 & 64 \\
\hline 8 & 2 & 1 & 135 & 118 & 92 & 4 & 63 & 62 \\
\hline 9 & 21 & 1 & 50 & 50 & 102 & 4 & 27 & 25 \\
\hline 10 & 21 & 1 & 25 & 25 & 112 & 4 & 64 & 65 \\
\hline 11 & 21 & 1 & 131 & 120 & 122 & 4 & 69 & 76 \\
\hline 12 & 21 & 1 & 98 & 94 & 132 & 4 & 53 & 48 \\
\hline 13 & 21 & 1 & 27 & 16 & 142 & 4 & 36 & 29 \\
\hline 14 & 2 & 1 & 39 & 35 & 152 & 4 & 24 & 27 \\
\hline 15 & 2 & 1 & 75 & 70 & 162 & 4 & 37 & 52 \\
\hline 16 & 21 & 1 & $<25$ & 8 & 172 & 4 & 18 & 28 \\
\hline 17 & 2 & 1 & $<22$ & 11 & 182 & 4 & 18 & 27 \\
\hline 18 & 2 & 1 & 46 & 52 & 02 & $\mathbf{5}$ & 139 & 145 \\
\hline 0 & 2 & 2 & 39 & 52 & 12 & 5 & 93 & 90 \\
\hline 1 & 2 & 2 & 32 & 35 & 22 & 5 & 124 & 123 \\
\hline 2 & 2 & 2 & 62 & 67 & 32 & $\mathbf{5}$ & 35 & 36 \\
\hline 3 & 2 & 2 & 225 & 225 & 42 & $\mathbf{5}$ & 154 & 160 \\
\hline 4 & 2 & 2 & 17 & 28 & 52 & $\mathbf{5}$ & 99 & 103 \\
\hline 5 & 2 & 2 & 70 & 68 & 62 & $\mathbf{5}$ & 26 & 28 \\
\hline 6 & 2 & 2 & 43 & 41 & 72 & 5 & 38 & 43 \\
\hline 7 & 2 & 2 & 90 & 76 & 82 & $\mathbf{5}$ & 27 & 32 \\
\hline 8 & 28 & 2 & 130 & 110 & 92 & $\mathbf{5}$ & 44 & 41 \\
\hline 9 & 2 & 2 & $<25$ & 5 & 102 & 5 & 44 & 45 \\
\hline 10 & 2 & 2 & 54 & 52 & 112 & 5 & 90 & 91 \\
\hline 11 & 2 & 2 & 50 & 47 & 122 & $\mathbf{5}$ & 127 & 136 \\
\hline 12 & 2 & 2 & $<28$ & 8 & 132 & 5 & $<25$ & 19 \\
\hline 13 & 2 & 2 & 163 & 169 & 142 & $\mathbf{5}$ & 125 & 114 \\
\hline 14 & 2 & 2 & $<27$ & 13 & 152 & $\mathbf{5}$ & 70 & 71 \\
\hline 15 & 2 & 2 & $<26$ & 6 & 162 & 5 & 66 & 75 \\
\hline 16 & 2 & 2 & $<25$ & 20 & 02 & 6 & 26 & 20 \\
\hline 17 & 2 & 2 & $<22$ & 20 & 12 & 6 & 107 & 98 \\
\hline 18 & 2 & 2 & 44 & 53 & 22 & 6 & 59 & 61 \\
\hline 0 & 2 & 3 & $<18$ & 15 & 32 & 6 & 91 & 87 \\
\hline 1 & 2 & 3 & 132 & 148 & 42 & 6 & 95 & 95 \\
\hline 2 & 2 & 3 & 116 & 131 & 52 & 6 & 39 & 45 \\
\hline 3 & 2 & 3 & 19 & 21 & 62 & 6 & 51 & 52 \\
\hline 4 & 2 & 3 & 40 & 45 & 72 & 6 & 95 & 99 \\
\hline
\end{tabular}

Acta Chem. Scand. 19 (1965) No. 10 


\begin{tabular}{|c|c|c|c|c|c|c|c|c|}
\hline$h k$ & $l$ & $\boldsymbol{F}_{\text {obs }}$ & $F_{\text {calc }}$ & $h$ & $k$ & $l$ & $F_{\text {obs }}$ & $F_{\text {calc }}$ \\
\hline 82 & 6 & 117 & 110 & & 2 & 8 & 26 & 25 \\
\hline 92 & 6 & 103 & 106 & 4 & 2 & 8 & 133 & 115 \\
\hline 102 & 6 & 42 & 36 & 5 & 2 & 8 & 65 & 56 \\
\hline 112 & 6 & $<26$ & 7 & 6 & 2 & 8 & 151 & 125 \\
\hline 122 & 6 & 42 & 50 & 7 & 2 & 8 & 45 & 45 \\
\hline 132 & 6 & 73 & 70 & 8 & 2 & 8 & 36 & $\mathbf{3 3}$ \\
\hline 142 & 6 & $<21$ & 3 & 9 & 2 & 8 & 57 & 50 \\
\hline 152 & 6 & 34 & 31 & 10 & 2 & 8 & 125 & 115 \\
\hline 02 & 7 & 27 & 29 & 11 & 2 & 8 & $<18$ & 20 \\
\hline 12 & 7 & 93 & 84 & 12 & 2 & 8 & 31 & 43 \\
\hline 22 & 7 & $<28$ & 5 & 0 & 2 & 9 & 115 & 106 \\
\hline 32 & 7 & 114 & 103 & 1 & $\overline{2}$ & 9 & 23 & 22 \\
\hline 42 & 7 & 27 & 30 & 2 & 2 & 9 & $<23$ & 1 \\
\hline 52 & 7 & 39 & 40 & 3 & 2 & 9 & $<23$ & 2 \\
\hline 62 & 7 & 27 & 29 & 4 & 2 & 9 & 66 & 51 \\
\hline 72 & 7 & 27 & 25 & 5 & 2 & 9 & 139 & 123 \\
\hline 82 & 7 & 26 & 21 & 6 & 2 & 9 & 32 & 36 \\
\hline 92 & 7 & 47 & 53 & 7 & 2 & 9 & $<20$ & 9 \\
\hline 102 & 7 & 34 & 31 & 8 & 2 & 9 & 46 & 47 \\
\hline 112 & 7 & 39 & 38 & 0 & 21 & 10 & 121 & 116 \\
\hline 122 & 7 & $<21$ & 16 & 1 & 21 & 10 & 27 & 29 \\
\hline 132 & 7 & 111 & 117 & 2 & 21 & 10 & 26 & 23 \\
\hline 02 & 8 & 126 & 113 & 3 & 21 & 10 & 82 & 88 \\
\hline 12 & 8 & 50 & 49 & 4 & 21 & 10 & 75 & 76 \\
\hline 22 & 8 & 26 & 16 & & & & & \\
\hline
\end{tabular}

The pentagonal bipyramid is quite regular as is shown in Fig. 3. The five anion-anion distances corresponding to edge sharing between the octahedra and the pentagonal bipyramid are all rather short, varying between 2.42 and $2.52 \AA$. The remaining anion-anion distances within the pentagonal bipyramid are 2.87-2.89 $\AA$. The distances between the non-metal atoms of the octahedra range from 2.55 to $3.08 \AA$. The metal-anion distances in the pentagonal bipyramid (1.98-2.14 $\AA$ ) have a mean value of $2.06 \AA$ while the corresponding distances of the three crystallographically different types of octahedra are $1.87-2.06 \AA, 1.90-2.14 \AA$, and $1.89-2.01 \AA$, with mean values of $1.95,1.99$, and $1.96 \AA$, respectively. These values agree well with the average niobiumoxygen distance of $1.99 \AA$ reported for the octahedra of $\alpha-\mathrm{Nb}_{2} \mathrm{O}_{5}{ }^{5}$

Sevenfold coordination has earlier been found for niobium in the $\mathrm{NbF}_{7}{ }^{2-}$ ion, ${ }^{6}$ in $\mathrm{NbOF}_{6}{ }^{3-}$ ion, ${ }^{7}$ in $\mathrm{NaNb}_{3} \mathrm{O}_{8},{ }^{8}$ and also in $0.4 \mathrm{Nb}_{2} \mathrm{O}_{5}-0.6 \mathrm{WO}_{3} .{ }^{9,10}$

In the structure of ScOF, as determined by Holmberg, ${ }^{11}$ the fluorine and oxygen atoms have been found to be ordered. This was shown by a leastsquares refinement of the structure. It is obviously in agreement with Pauling's Electrostatic Valence Principle. ${ }^{12}$ If $\sum s_{\mathrm{i}}$ (the sum of the strength of the electrostatic valence bonds) is calculated according to Pauling for the different twofold anion positions in $\mathrm{LiNb}_{6} \mathrm{O}_{15} \mathrm{~F}$, the lowest positive potential is obtained for the anion position which is shared between two pentagonal bipyramids in the point position $2(f)$ and has the coordinates $\frac{1}{4}, \frac{1}{2}, 0.69$. 
Table 4. Interatomic distances in a building block of the $\mathrm{LiNb}_{6} \mathrm{O}_{15} \mathrm{~F}$ structure (Fig. 3).

\begin{tabular}{|c|c|c|}
\hline $\begin{array}{l}2 \mathrm{Nb}_{1} \\
2 \mathrm{Nb}_{1} \\
\mathrm{Nb}_{1}\end{array}$ & $\begin{array}{l}-\mathbf{N b}_{\mathbf{3}} \\
=\mathbf{N b _ { 3 }} \\
=\mathbf{N} b_{4}\end{array}$ & $\begin{array}{l}3.26 \pm 0.001 \\
3.37 \pm 0.001 \\
3.29 \pm 0.001\end{array}$ \\
\hline $\begin{array}{ll}1 & \mathrm{Nb}_{1} \\
2 & \mathrm{Nb}_{1} \\
2 & \mathrm{Nb}_{1} \\
2 & \mathrm{Nb}_{1}\end{array}$ & $\begin{array}{l}-O_{1} \\
-O_{2} \\
-O_{3} \\
-O_{1}(\equiv F)\end{array}$ & $\begin{array}{l}2.04 \pm 0.04 \\
2.08 \pm 0.03 \\
2.14 \pm 0.02 \\
1.98 \pm 0.001\end{array}$ \\
\hline $\begin{array}{ll}1 & \mathrm{Nb}_{2} \\
1 & \mathrm{Nb}_{2} \\
1 & \mathrm{Nb}_{2} \\
1 & \mathrm{Nb}_{2} \\
2 & \mathrm{Nb}_{2}\end{array}$ & $\begin{array}{l}-O_{1} \\
=O_{2} \\
=O_{5} \\
=O_{8} \\
=O_{7}\end{array}$ & $\begin{array}{l}2.06 \pm 0.01 \\
1.93 \pm 0.03 \\
1.87 \pm 0.03 \\
1.90 \pm 0.03 \\
1.98 \pm 0.001\end{array}$ \\
\hline $\begin{array}{l}1 \mathrm{Nb}_{3} \\
1 \\
\mathrm{Nb}_{3} \\
1 \mathrm{Nb}_{3} \\
1 \mathrm{Nb}_{3} \\
2 \mathrm{Nb}_{3}\end{array}$ & 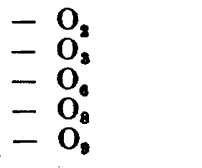 & $\begin{array}{l}2.14 \pm 0.03 \\
2.01 \pm 0.03 \\
1.90 \pm 0.03 \\
1.92 \pm 0.003 \\
1.98 \pm 0.001\end{array}$ \\
\hline $\begin{array}{l}\mathrm{Nb}_{2} \\
2 \mathrm{Nb} \\
\mathrm{Nb}_{4}\end{array}$ & $\begin{array}{l}=\mathbf{O}_{2} \\
=\mathbf{O}_{5} \\
=\mathbf{O}_{10}\end{array}$ & $\begin{array}{l}2.01 \pm 0.02 \\
1.89 \pm 0.03 \\
1.98 \pm 0.001\end{array}$ \\
\hline $\begin{array}{ll}2 & O_{1} \\
2 & O_{2} \\
1 & O_{3}\end{array}$ & $\begin{array}{l}-O_{2} \\
=O_{3} \\
-O_{3}\end{array}$ & $\begin{array}{l}2.42 \pm 0.03 \\
2.48 \pm 0.03 \\
2.52 \pm 0.05\end{array}$ \\
\hline $\begin{array}{ll}2 & \mathrm{O}_{1} \\
4 & \mathrm{O}_{2} \\
4 & \mathrm{O}_{3}\end{array}$ & $\begin{array}{r}-O_{4}(\equiv \mathbf{F}) \\
-O_{1}(\equiv \mathbf{F}) \\
-O_{0}(\equiv \mathbf{F})\end{array}$ & $\begin{array}{l}2.87 \pm 0.001 \\
2.89 \pm 0.03 \\
2.89 \pm 0.02\end{array}$ \\
\hline $\begin{array}{ll}1 & O_{1} \\
2 & O_{1} \\
1 & O_{2} \\
2 & O_{2} \\
1 & O_{5} \\
2 & O_{5} \\
2 & O_{6}\end{array}$ & $\begin{array}{l}-o_{8} \\
=O_{7} \\
=o_{6} \\
=O_{7} \\
=o_{6} \\
=o_{7} \\
=O_{7}\end{array}$ & $\begin{array}{lll}2.74 & \pm 0.04 \\
2.88 & \pm 0.03 \\
\mathbf{3 . 0 7} & \pm 0.04 \\
\mathbf{2 . 7 5} & \pm 0.03 \\
\mathbf{2 . 6 9} & \pm 0.04 \\
\mathbf{2 . 7 5} & \pm 0.03 \\
\mathbf{2 . 7 3} & \pm 0.03\end{array}$ \\
\hline $\begin{array}{ll}1 & O_{2} \\
2 & O_{2} \\
1 & O_{3} \\
2 & O_{3} \\
1 & O_{6} \\
2 & O_{6} \\
2 & O_{8}\end{array}$ & $\begin{array}{l}=0_{0} \\
=0_{9} \\
=O_{9} \\
=0_{8} \\
=0_{9} \\
=0_{9}\end{array}$ & 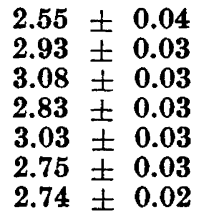 \\
\hline $\begin{array}{ll}2 & O_{3} \\
4 & O_{3} \\
1 & O_{5} \\
4 & O_{5}\end{array}$ & $\begin{array}{l}-O_{s} \\
=o_{10} \\
=O_{s} \\
=O_{10}\end{array}$ & $\begin{array}{l}2.80 \pm 0.03 \\
2.79 \pm 0.03 \\
2.88 \pm 0.05 \\
2.76 \pm 0.03\end{array}$ \\
\hline
\end{tabular}

Mean value 
As for the arrangement of the lithium atoms, two possible positions in the unit-cell, $2(d)$ or $4(j)$ with the approximate coordinates $0.08, \frac{1}{2}, 0.10$, present themselves as likely ones. The $4(j)$ position requires a random distribution of the two lithium atoms. Both positions would give rise to six to eight lithiumanion contacts at distances ranging from 2.5 to $3.0 \AA$.

The same type of building blocks, as are present in $\mathrm{LiNb}_{6} \mathrm{O}_{15} \mathrm{~F}$, viz. one pentagonal bipyramid sharing edges with five octahedra, has previously been found in the structures of $\mathrm{W}_{18} \mathrm{O}_{49},{ }^{13,14} \mathrm{Mo}_{17} \mathrm{O}_{47},{ }^{14,15}$ and $\mathrm{Mo}_{5} \mathrm{O}_{14 \cdot}{ }^{14,16}$ The building blocks in $\mathrm{W}_{18} \mathrm{O}_{49}$ and $\mathrm{Mo}_{17} \mathrm{O}_{47}$ are, however, joined together both by corners and by edges. Also in contrast to $\mathrm{LiNb}_{6} \mathrm{O}_{15} \mathrm{~F}$ all these binary compounds contain additional octahedra, which are not coupled to the pentagonal bipyramid.

Acknowledgements. The author wishes to thank Professor Arne Magnéli for his interest in this work and his valuable comments on the manuscript and also Dr. Sten Andersson for having suggested this investigation.

This work has taken place within a research program supported by the Swedish Natural Science Research Council.

\section{REFERENCES}

1. Lundberg, M. and Andersson, S. Acta Chem. Scand. 19 (1965) 1376.

2. Roth, R. S. J. Res. Natl. Bur. Std. 62 (1959) 27.

3. Werner, P. E. IUCr World List of Crystallographic Computer Programs (1962). Accession No. 6019.

4. Åsbrink, S. and Brändén, C. IUCr World List of Crystallographic Computer Programs (1962). Accession No. 6023.

5. Gatehouse, B. M. and Wadsley, A. D. Acta Cryst. 17 (1964) 1545.

6. Hoard, J. L. J. Am. Chem. Soc. 61 (1939) 1252.

7. Williams, M. B. and Hoard, J. L. Ibid. 64 (1942) 1139.

8. Andersson, S. Unpublished work.

9. Sleight, A. and Magnéli, A. Acta Chem. Scand. 18 (1964) 2007.

10. Sleight, A. To be published.

11. Holmberg, B. In print.

12. Pauling, L. J. Am. Chem. Soc. 51 (1929) 1010.

13. Magnéli, A. Arkiv Kemi 1 (1949) 223.

14. Kihlborg, L. Arkiv Kemi 21 (1963) 471.

15. Kihlborg, L. Acta Chem. Scand. 17 (1963) 1485.

16. Kihlborg, L. Arkiv Kemi 21 (1963) 427.

Received August 20, 1965. 